\title{
CONTUSION OF THE LUNG IN CHILDHOOD
}

BY

\author{
A. SCHWARTZ and J. B. BORMAN \\ From the Departments of Diagnostic Radiology and Thoracic Surgery, Hadassah University Hospital, \\ Jerusalem, Israel
}

(RECEIVED FOR PUBLICATION MARCH 24, 1961)

Chest injuries are on the increase in all civilized countries, mainly due to the rising number of traffic accidents (Zenker, 1956; Daughtry, 1957; Strug, Glass, Leon and Salatich, 1960).

The clinical picture of severe chest trauma, both penetrating (Gray, Harrison, Couves and Howard, 1960) and blunt (Harrison, Gray, Couves and Howard, 1960; Griffiths, 1960), has been amply described. Lesser injuries, however, have been neglected in the literature, although these cases often have radiological evidence of lung damage. This is particularly so in children, in whom the pulmonary changes may be overlooked because of the paucity of clinical symptoms. The purpose of this paper is to describe four cases of blunt chest injury in children and to discuss the symptomatology and radiological appearance of this condition.

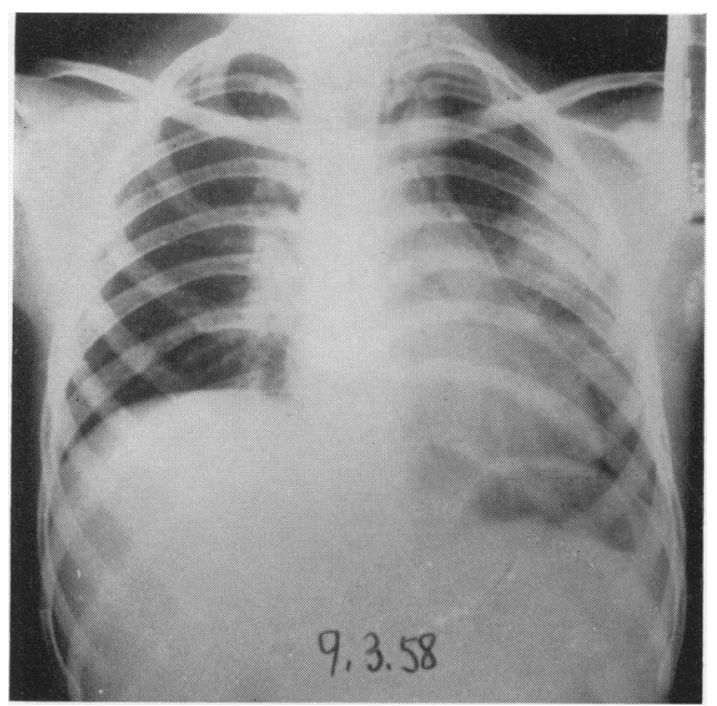

FIG. 1.-Case 1: Postero-anterior radiograph of the chest showing air in the mediastinum extending into the neck. Note the air outlining the left border of the descending aorta. Radiolucent area with fluid level visible in the opacity in the left middle lung field.

\section{Case Reports}

Case 1. An 8-year-old boy fell, head down, from a height of 5 metres. He was unconscious following the accident and on admission one and a half hours later was still semi-conscious. Temperature was $37^{\circ} \mathrm{C}$., pulse $130 / \mathrm{min}$. and respiration $44 / \mathrm{min}$. A skull radiograph showed no fracture, but air was noted in the soft tissues of the neck and a radiograph of the chest was obtained. This showed a pneumomediastinum and shadowing of the left mid-lung field with a rounded translucent area containing a fluid level (Fig. 1). No rib fractures were noted, and there was no pneumothorax and no fluid in the pleural cavity.

The diagnosis of contusion of the lung and pneumomediastinum was made. On antibiotic therapy the child's general condition improved. The shadow in the left lung resolved rapidly and when discharged eight days after his injury the only abnormal x-ray finding consisted of a residual ring shadow $2 \mathrm{~cm}$. in diameter (Fig. 2).

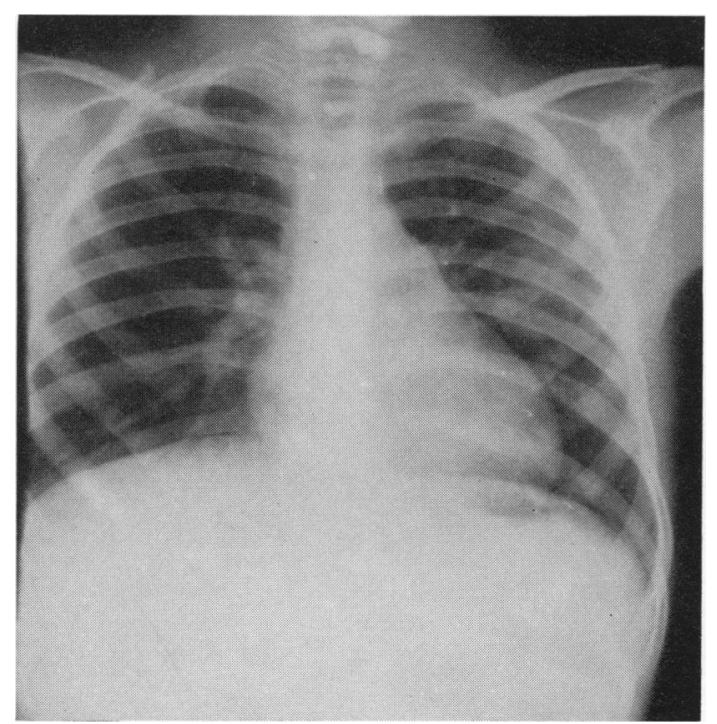

FIG. 2.-Case 1: Eight days later the only abnormal finding is a ring shadow, $2 \mathrm{~cm}$. in diameter, at the site of the previous opacity. 


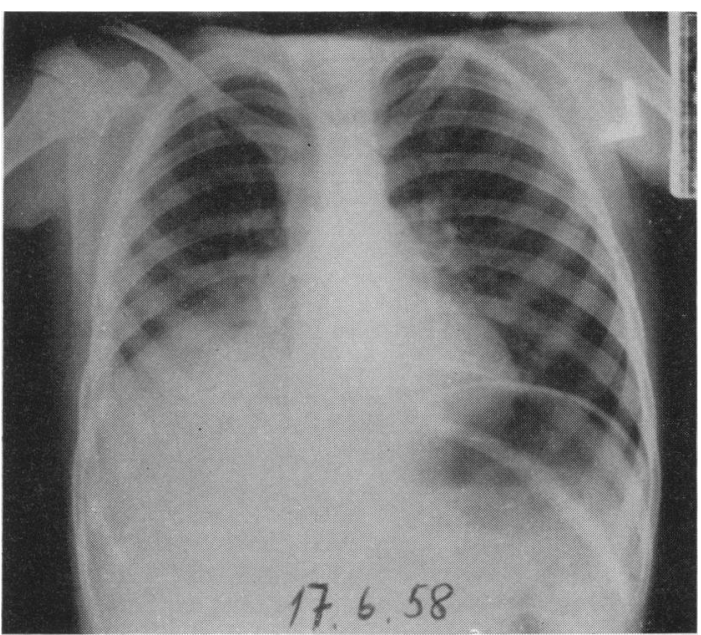

FIG. 3.-Case 2: Radiograph, taken on admission, showing a dense opacity obscuring the right diaphragm.

Case 2. An 8-year-old girl was admitted 24 hours after having been knocked down by a motor car. On admission she complained of pain in the right upper abdomen. She had no cough, haemoptysis or dyspnoea. Examination revealed a pale child with a normal blood pressure but with a pulse of $160 / \mathrm{min}$. and a temperature of $40^{\circ} \mathrm{C}$. Crepitations were heard over both lung bases. She had a bilateral Babinsky reflex. A skull radiograph was normal. A chest radiograph revealed a density in the right lower lung field obscuring the diaphragm. No rib fracture could be detected. The rest of the lung

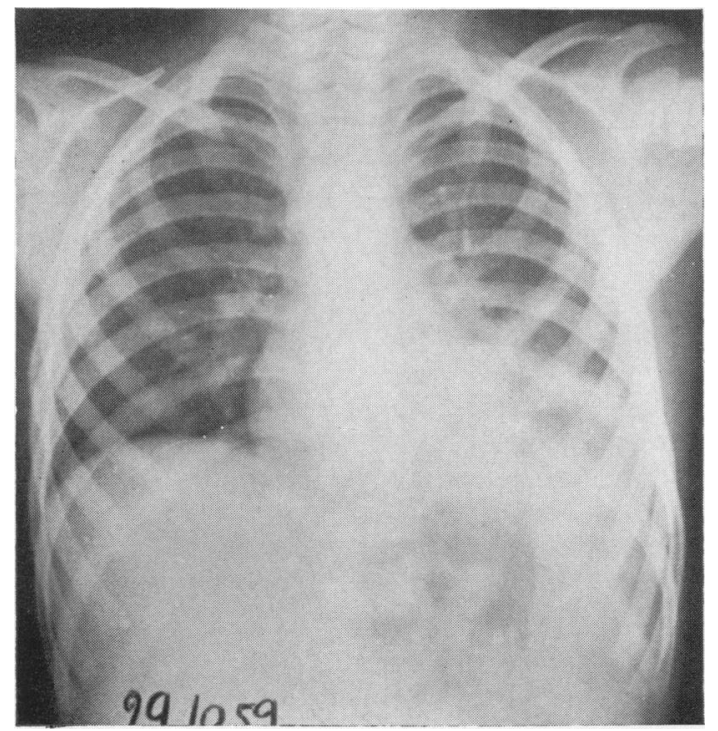

FIG. 5.-Case 3: Radiograph taken on admission showing haziness of the left lower lung field with blurring of the diaphragm.

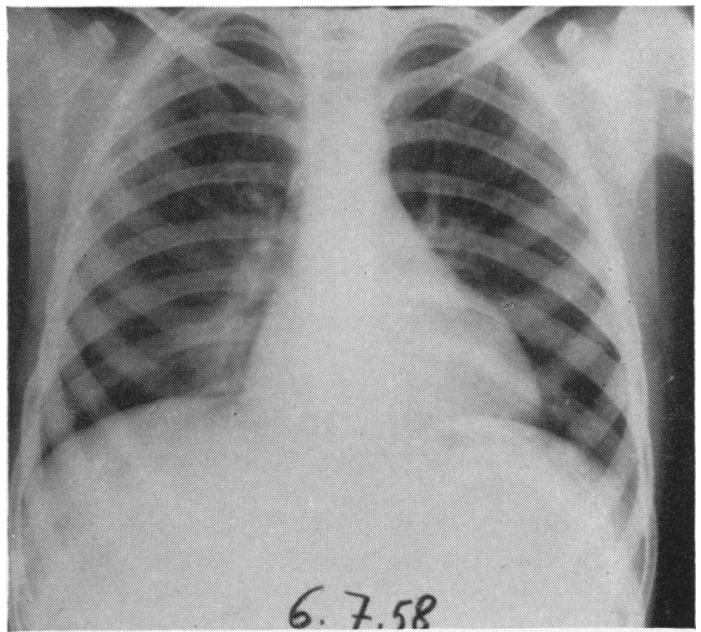

FIG. 4.-Case 2: Radiograph 19 days later shows minimal residual density adjacent to the right border of the heart.

fields were normal (Fig. 3). The opacity in the right chest was interpreted as contusion of the lung.

Treatment with penicillin and streptomycin resulted in a gradual decline of the fever accompanied by clearing of the lung. On discharge 19 days after injury the lung shadow had cleared almost completely (Fig. 4).

Case 3. A 4-year-old boy was admitted as an emergency following a fall on to his left chest. On examination he was pale, but not in distress. Blood pressure and respiration were normal, pulse $120 / \mathrm{min}$. and tem-

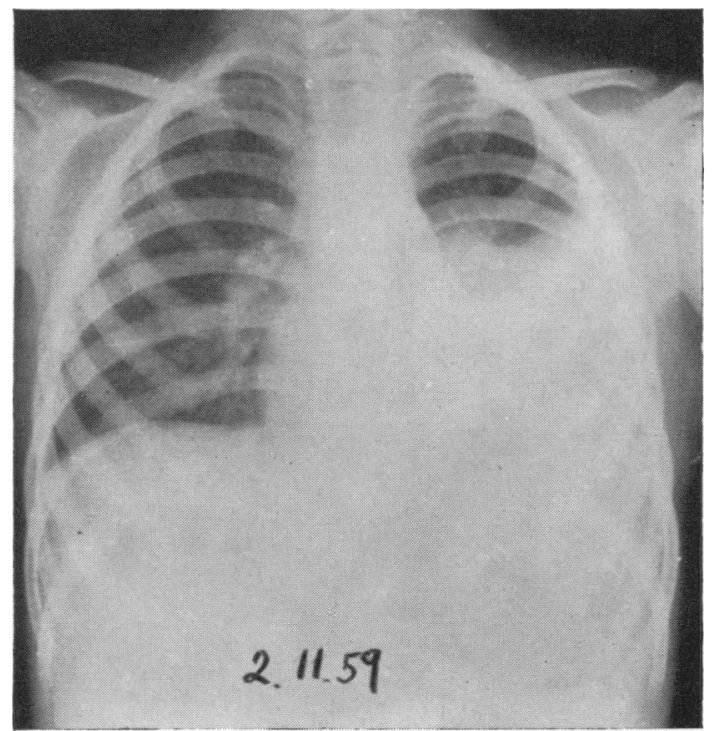

Fig. 6.-Case 3: Three days after admission the density, now occupying the lower half of the left hemithorax, is typical of intrapleural fluid. 


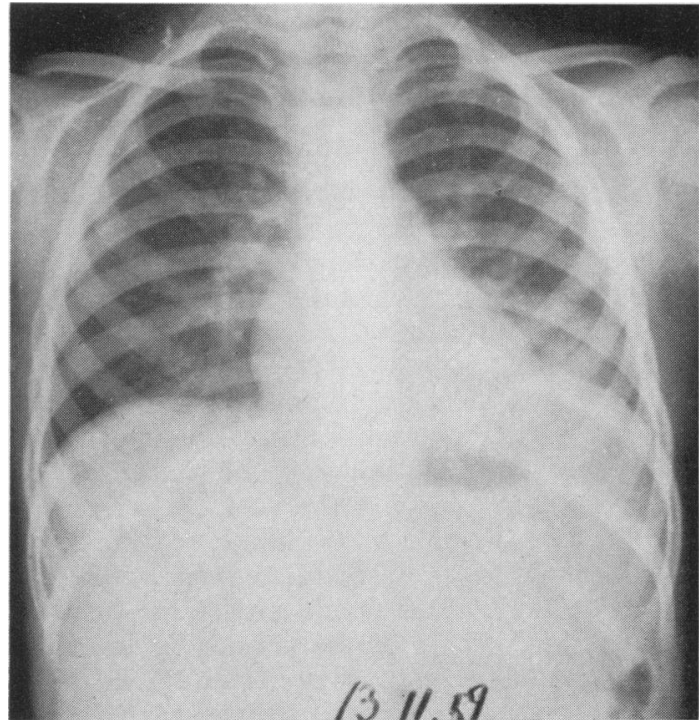

FIG. 7.-Case 3: Radiograph on the day of discharge shows only slight residual clouding at left base.

perature $37^{\circ}$ C. Dullness and diminished air entry were found at the left base.

Chest radiograph showed a broad belt of haziness in the left lower lung field obscuring the diaphragm. There was a suggestion of fluid in the left costophrenic sinus. No pneumothorax or rib fracture was detected (Fig. 5).

The diagnosis of contusion of the lung with haemothorax was made.

Antibiotic therapy was immediately commenced, but the temperature rose for the next three days. A chest film at this time showed a dense shadow occupying the lower half of the left hemithorax (Fig. 6). Pleural tap was done and $60 \mathrm{ml}$. of dark blood removed. A chest radiograph taken after this showed only slight reduction in the size of the shadow, suggesting that the greater part of it was due to intrapulmonary pathology.

The antibiotic therapy was continued and the temperature gradually settled. When discharged 16 days after admission the lungs, clinically and radiologically, had almost cleared (Fig. 7). The child remained symptom-free on follow-up examination one month after discharge.

Case 4. A 12-year-old boy was rushed to the emergency room after being sandwiched between two cars, and sustaining a crushing injury to his chest.

Examination revealed a frightened, pale boy, not in shock. Blood pressure was $125 / 70 \mathrm{~mm}$. $\mathrm{Hg}$, pulse $90 / \mathrm{min}$. and temperature $38^{\circ} \mathrm{C}$. No abnormal findings could be detected in the lungs. Tyre pressure marks were present on the anterior and posterior chest walls.

A radiograph of the chest showed a widened mediastinum with an ill-defined left border. There were nodular densities scattered throughout the left lung

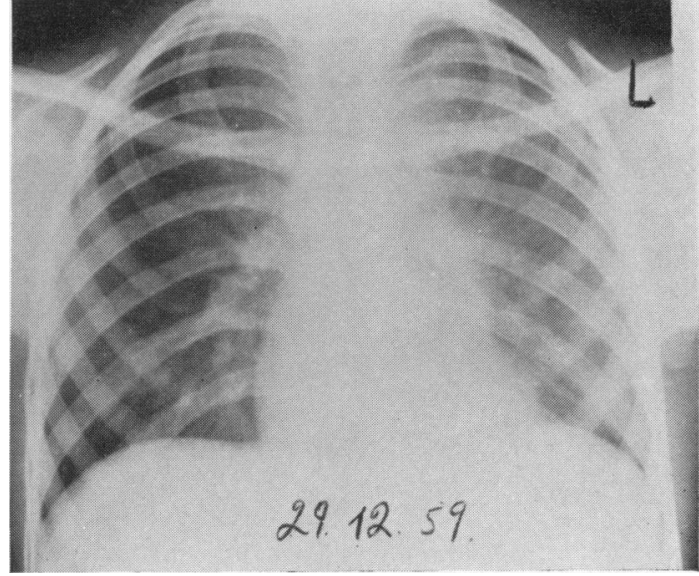

FIG. 8.-Case 4: Radiograph on admission showing widening of the mediastinum with blurring of its left border, small densities scattered in the left lung field and a tiny apical pneumothorax.

and a tiny left apical pneumothorax was noted (Fig. 8). No fracture of the ribs or sternum was detected.

Contusion of the lung with mediastinal haematoma was diagnosed. He received penicillin and streptomycin and the temperature returned to normal within three days. A chest film on the third day after admission showed increased mediastinal widening, fluid in the left pleural cavity and confluence of the nodular densities in the left lower lung field (Fig. 9). Subsequent radiographs showed gradual decrease of the mediastinal shadow, resorption of the fluid, clearing of the lung field and disappearance of the pneumothorax.

When discharged two weeks after his accident his chest was clinically and radiographically normal and remained so on follow-up examination two months later (Fig. 10).

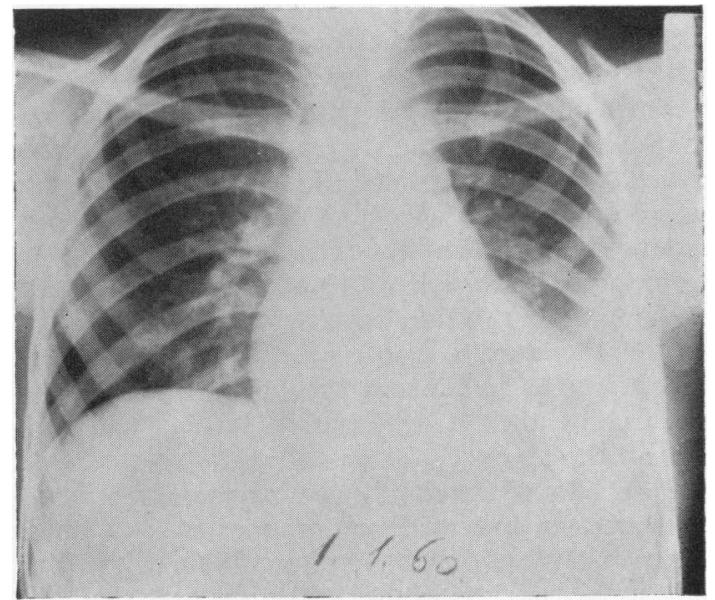

FIG. 9.-Case 4: Three days later the mediastinum is still wide, but its left border is now distinct. Fluid is present in the left pleural cavity. 


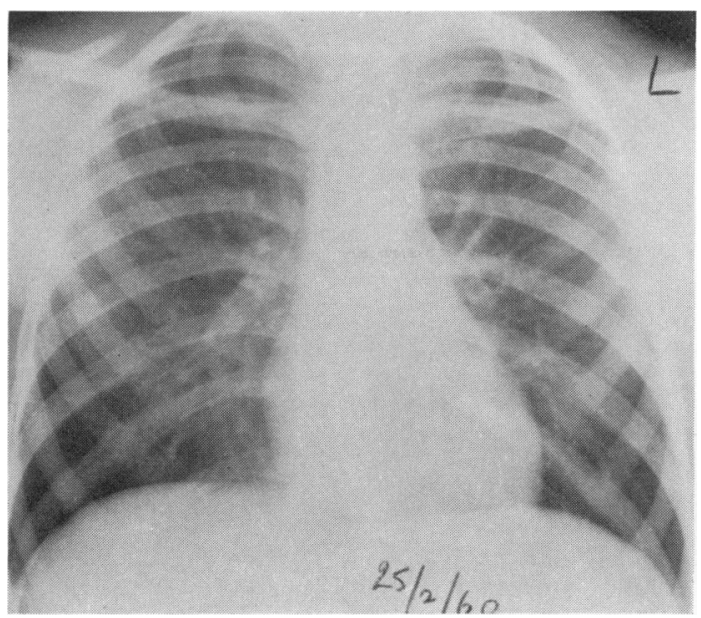

Fig. 10.-Case 4: Two months after injury the chest is normal.

\section{Discussion}

Blunt chest injury in children differs in its symptomatology from that occurring in adults. This is due to the elasticity of the thoracic cage in the former, which allows a blunt injury to contuse and even lacerate the lung and/or other intrathoracic organs without fracturing a rib (Wilson, 1943). Because of the absence of a rib fracture the injured child may not complain of pain in the chest, and consequently a chest radiograph is often omitted. The clinical signs of injury to the lung being minimal, or even absent, attention may be drawn to a concomitant injury. Two of our cases were admitted because of head injuries. In one of them a chest radiograph was not requested on admission (Case 1) and a pulmonary injury was only detected by the presence of air in the soft tissues of the neck as seen on the skull radiograph. Haemoptysis, dyspnoea and cough, the expected findings accompanying lung damage, were absent in our cases. This is at variance with the findings of Sealy (1949) who found haemoptysis in 35 out of 40 adults with contusion of the lung due to non-penetrating injury.

It is therefore a good rule to obtain a chest radiograph if possible in the upright position, of every child after a deceleration type of accident, even in the absence of signs of injury to the chest. Radiographs of the chest in our cases did not reveal rib fractures, but evidence of pulmonary damage was seen in all of them. Fracture of a rib, however, is probably an important indication that the child has received a severe blow. During the period of time the four cases were observed, two other children were admitted with fractured ribs as a result of traffic accidents. Both of them had extensive haemopneumothorax with contusion of the lung. Interestingly, they, too, had no cough or haemoptysis despite the severe trauma to the lung.

The radiographic changes after a blunt injury without rib fracture are variable and depend upon the intrathoracic organ injured. If only the lung is injured, as in these cases, fuzzy opacities of different size and density with irregular borders are the principal findings (Westermark, 1941). A pneumothorax or haemothorax or both may be present. The mediastinum is occasionally widened and pneumomediastinum may be evident. Subcutaneous emphysema in the neck and chest wall may be seen with, or without, pneumothorax (Vandooren, 1958). Extensive, increasing subcutaneous emphysema should arouse the suspicion of a tear or rupture of the trachea or bronchus. A high diaphragm on the injured side and dilatation of the stomach is of common occurrence (Virshup, 1960).

The underlying pathology causing the intrapulmonary opacities is still uncertain. The possible causes of the lung shadows appearing in the radiograph are interstitial haematoma (Major, 1956), acute localized oedema (Zenker, 1956; Barrett, 1960), patchy confluent atelectasis (Major, 1956), and intra-alveolar haemorrhage (Sealy, 1949). Probably all the above factors play some part in the production of the lung opacities.

These pulmonary contusions, per se, do not require surgical intervention. Treatment should be directed primarily towards concomitant injuries if present, e.g. multiple fractures, rupture of spleen or other abdominal viscera and head injuries. Children with lung contusion alone should receive prophylactic antibiotic therapy, as infection may complicate these injuries (Fallon, 1940; Phillips, 1947). They should be kept under observation in hospital until the lungs are clear. This generally occurs within three weeks (Sealy, 1949; Westermark, 1941; Greening, Kynette and Hodes, 1957).

Our cases had some fever during the first few days, but on antibiotic treatment the temperature settled. None of them developed frank pneumonia, abscess or bronchiectasis and the final follow-up examination showed clinically and radiologically normal lungs. Cases have been described which developed round or ring shadows persisting for as long as three months (Greening et al., 1957). Pulmonary haematoma due to blunt injury may occasionally persist even longer and masquerade as a coin lesion. Such cases have undergone thoracotomy (Salyer, Blake and Forsee, 1953). 


\section{Summary}

The clinical picture and radiological appearance of lung contusion in children after blunt injuries to the chest is described. Illustrative cases are presented.

There is a discrepancy between the minimal clinical findings and the marked $x$-ray changes of the lung.

A chest film should be obtained of all children involved in deceleration accidents even in the absence of symptoms and signs pointing to chest injury.

\section{REFERENCES}

Barrett, N. R. (1960). Early treatment of stove-in chest. Lancet, 1,293

Daughtry, D. C. (1957). Management of nonpenetrating thoracic injuries. Amer. Surg., 23, 462.

Fallon, M. (1940). Lung injury in the intact thorax: with report of a case. Brit. J. Surg., $28,39$.

Gray, A. R., Harrison, W. H., Jr., Couves, C. M. and Howard, J. M. (1960). Penetrating injuries to the chest. Clinical results in the management of 769 patients. Amer. J. Surg., 100, 709.
Greening, R., Kynette, A. and Hodes, P. J. (1957). Unusual pulmonary changes secondary to chest trauma. Amer. J. Roentgenol., 77, 1059 .

Griffiths, H. W. C. (1960). Crush injuries of the chest. J. roy. Coll. Surg. Edinb. 6, 13 .

Harrison, W. H., Jr. Gray, A. R., Couves, C. M. and Howard, J. M (1960). Severe non-penetrating injuries to the chest. Clinical results in the management of 216 patients. Amer. J. Surg. results in the management of 216 patients. Amer. J. Surg., 100,715 .

Major, H. (1956). Der posttraumatische Lungenkollaps. Arch. klin. Chir., 284, 177.

Phillips, E. (1947). Pneumonia following nonpenetrating pulmonary injuries. J. Amer. med. Ass., 133, 161.

Salyer, J. M., Blake, H. A. and Forsee, J. H. (1953). Pulmonary hematoma. J. thorac. Surg., 25, 336.

Sealy, W. C. (1949). Contusions of the lung from nonpenetrating injuries to the thorax. Arch. Surg. (Chicago), 59, 882.

Strug, L. H., Glass, B., Leon, W. and Salatich, M. (1960). Severe crushing injuries of the chest. A simple method of stabilization. J. thorac. Surg., 39, 166.

Vandooren, M. (1958). Reflexions sur trois contusions thoraciques graves. Poumon, 14, 173 .

Virshup, M. (1960). The anteriorly crushed chest or 'steering-wheel injury'. $f$, thorac. Surg, 39, 579.

Westermark, N. (1941). A roentgenological investigation into traumatic lung changes arisen through blunt violence to the thorax. Acta radiol., 22, 331 .

Wilson, J. V. (1943). The pathology of closed injuries of the chest. Brit. med. J., 1, 470.

Zenker, R. (1956). Die geschlossenen und offenen Verletzungen der Lunge und des Brustfells. Arch. klin. Chir., 284, 152. 